\title{
Spectral Studies of Some Hydroxy-derivatives of Anthraquinones
}

By M. S. El Ezaby, T. M. Salem,* A. H. Zewail, and R. Issa, Inorganic Section, Chemistry Department, Faculty of Science, Alexandria University, Alexandria, U.A.R.

The U.v., visible, and i.r. spectra of several hydroxy-anthraquinones are discussed and the bands are assigned. These bands are compared with those of anthracene and anthraquinone. The band at $207 \mathrm{~nm}$. is assigned to a $n-\sigma^{*}$ transition: the bands at 252,272 , and $326 \mathrm{~nm}$. are assigned by measuring spectra in solvents of various polarities. The stability constant for the 1,2-dihydroxyanthraquinone-ethanol complex is obtained.

THERE have been few investigations on the electronic spectra of quinones..$^{1-5}$ Morton and Earlam ${ }^{1}$ showed that the position and type of substituents had a considerable effect on the absorption spectra, and Sheppard and Newsome ${ }^{2}$ showed that the spectra were affected by the polarity of the solvent. Peters and Sumner ${ }^{3}$ discussed the changes in spectra in terms of the electromeric effects of substituents in anthraquinones; the difference in the i.r. spectra of these compounds in sulphuric acid and oleum showed that they had a similar electronic structure in these solvents. Yoshida and Takabayashi ${ }^{4}$ assigned all the bands in the spectra of anthraquinone and some derivatives to $\pi-\pi^{*}$ transitions.

We have investigated the effect of the medium on the electronic transition of some di-, tri-, and tetra-hydroxyanthraquinones. The effect on charge-transfer bands and on complex formation is also discussed. Stability constants for the complexes were determined where possible.

\section{EXPERIMENTAL}

1,4- (I) and 1,5-Dihydroxyanthraquinone (II) were prepared by the methods of Gatterman and Wielanel, ${ }^{5}$ and Schwenk. $^{6}$ 1,2-Dihydroxy- (III), 1,2,4-trihydroxy- (IV), and 1,4,5,8-tetrahydroxy-anthraquinone (V) (B.D.H. reagents) were purified by recrystallisation or sublimation. Satisfactory analyses and m.p.s. were obtained. U.v., visible, and i.r. spectra were recorded with Unicam SP 800 and SP 200 instruments, respectively.

\section{RESULTS AND DISCUSSION}

On a localised picture, the hydroxy-anthraquinone should have characteristic bands due to electronic transitions of both the substituent and the anthraquinone nucleus. Since the transition energy of the substituent is usually high, ${ }^{7}$ we have not considered this transition. The anthraquinone part of the molecule can be regarded as a 9,10-disubstituted anthracene; hence a comparison of the absorption spectrum of anthraquinone with that of anthracene could help in the assignment of bands corresponding to the quinonoid and benzenoid systems.

The absorption spectrum of anthraquinone in ethanol (Figure 1) has peaks at 207, 252, 272, and $326 \mathrm{~nm}$. The peaks at 252,272 , and $326 \mathrm{~nm}$. have been assigned

1 R. A. Morton and W. T. Earlam, J. Chem. Soc., 1941, 159.

2 S. E. Sheppard and P. T. Newsome, J. Amer. Chem. Soc., 1942, 64, 2937.

${ }^{3}$ R. H. Peters and H. H. Sumner, J. Chem. Soc., 1953, 2101.

4 Z. Yoshida and F. Takabayashi, Tetrahedron, 1968, 24, 933. previously to $\pi-\pi *$ transitions. ${ }^{4}$ The lower intensity of the $207 \mathrm{~nm}$. band compared with that of the $252 \mathrm{~nm}$.<smiles>CCCCC(=O)c1c(O)cccc1C(=O)O</smiles>

(I)<smiles>O=C1C(=O)C(O)=C(O)C(O)=C1O</smiles>

(IV)<smiles>O=C1c2ccccc2C(=O)c2ccccc21</smiles>

(D)<smiles>O=C1c2ccccc2C(=O)c2c(O)cccc21</smiles><smiles>O=C1c2cccc(O)c2C(=O)c2c(O)cccc21</smiles>

(Y)

band and the blue shift as the polarity of the solvent increases (Figure 2), could be due to blocking of the carbonyl lone pair, so the $207 \mathrm{~nm}$. band was assigned to a $n-\sigma^{*}$ transition. The $252 \mathrm{~nm}$. band is present in the spectra of anthracene and anthraquinone; it was assigned, therefore, to a $\pi-\pi *$ transition of the benzenoid

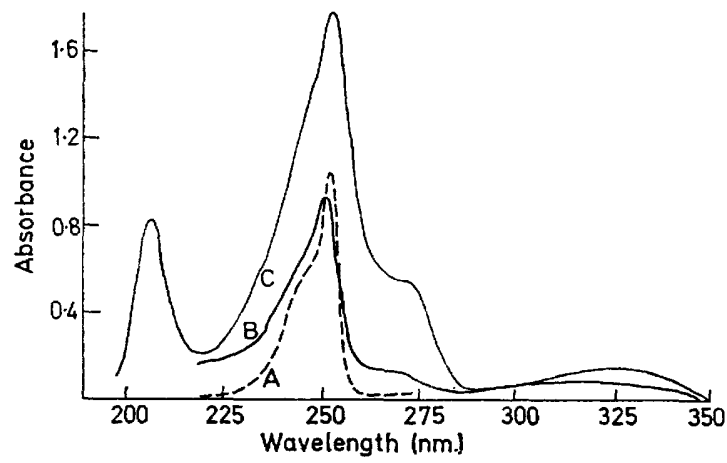

FIGURE 1 Absorption spectra of anthracene (A), anthraquinone in ethanol (B), and anthraquinone in ether (C)

system. The 272 and $326 \mathrm{~nm}$. bands are absent from the spectrum of anthracene; hence they were assigned to quinonoid $\pi-\pi^{*}$ transitions. They were further assigned to transition of the ethylenic and carbonyl system by measuring the spectra in solvents of various polarities.

The u.v. spectra of the hydroxy-compounds in ether (low polarity) (Figure 2) show separate bands due to the carbonyl and ethylenic systems, as in anthraquinone. Also, the 207 band shows a blue shift in more polar

5 L. Gatterman and $\mathrm{H}$. Wieland, 'Laboratory Methods of Organic Chemistry,' Macmillan, London, 1946.

6 E. Schwenk, J. prakt. Chem., 1921, 108, 106.

7 J. N. Murrell, The Theory of the Electronic Spectra of Organic Molecules,' Methuen, London, 1963. 


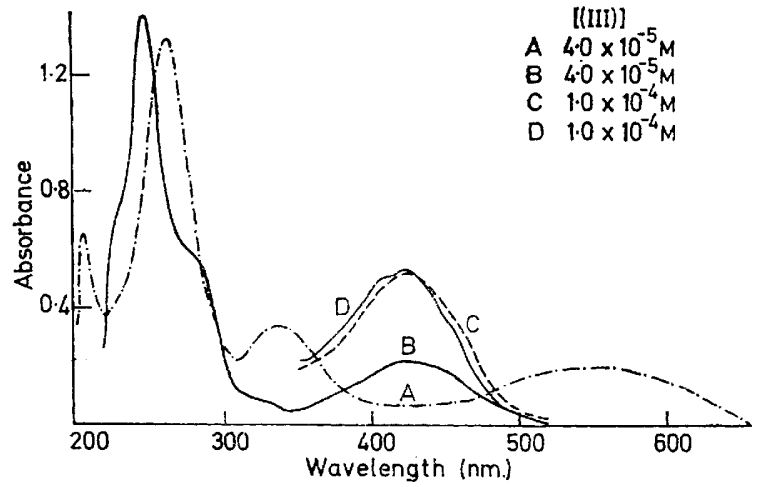

FIGURE 2 Absorption spectra of 1,2-dihydroxyanthracene (III) in ethanol (A), ether (B), acetone (C), and chloroform (D)

solvents. In chloroform, this may be due to a dipoledipole interaction with the non-hydrogen bonded hydroxy-group [structure (A)].<smiles></smiles>

The compounds (especially those with a non-hydrogenbonded hydroxy-group) show a red shift (compared with 1,2-dihydroxyanthraquinone, is almost absent from the spectra of 1,4-di-, 1,5-di-, 1,2,4-tri-, and 1,4,5,8-tetrahydroxyanthraquinone. In the latter compounds

TABLE 1

Molar extinction coefficients $\left(\times 10^{-4}\right)$ of the hydroxyanthraquinones at different wavelengths

\begin{tabular}{|c|c|c|c|c|c|c|c|}
\hline \multirow[b]{2}{*}{ Compound } & \multicolumn{3}{|c|}{ Ether } & \multicolumn{4}{|c|}{ Ethanol } \\
\hline & 252 & 275 & 326 & 210 & 252 & 275 & 326 \\
\hline (III) & $3 \cdot 20$ & 1.55 & $0 \cdot 25$ & 1.45 & $2 \cdot 70$ & $2 \cdot 50$ & 0.80 \\
\hline & $5 \cdot 70$ & 1.83 & 0.33 & $1 \cdot 3$ & $2 \cdot 10$ & $0 \cdot 80$ & 0.2 \\
\hline$(\mathrm{II})$ & $2 \cdot 37$ & $1 \cdot 27$ & 0.07 & 0.60 & 0.68 & 0.40 & $0 \cdot 1$ \\
\hline (IV) & $2 \cdot 53$ & 0.77 & $0 \cdot 30$ & $1 \cdot 10$ & $0 \cdot 76$ & 1.24 & 0.4 \\
\hline (V) & - & - & - & 0.80 & 0.90 & 0.74 & 0.32 \\
\hline
\end{tabular}

this may be due to blocking of the $\pi$ electrons of the carbonyl groups, through hydrogen-bonding. However, the quinonoid bonds of the hydroxy-compounds in ether are nearly the same as those of anthraquinone. The proton acceptor nature of ethanol weakens the hydrogen bond between the hydroxy and carbonyl groups, so the transition energy of the $n-\sigma^{*}$ and $\pi-\pi *$ bands is changed.

The red shift of the quinonoid bands in ethanol may be due to dissociation, which increases delocalisation through the quinonoid system. This is shown clearly in 1,2-di-, 1,2,4-tri-, and 1,4,5,8-tetra-hydroxyanthraquinone (Table 1) which have free hydroxy-groups. At high $\mathrm{pH}$, conjugation of the carbonyl group with the

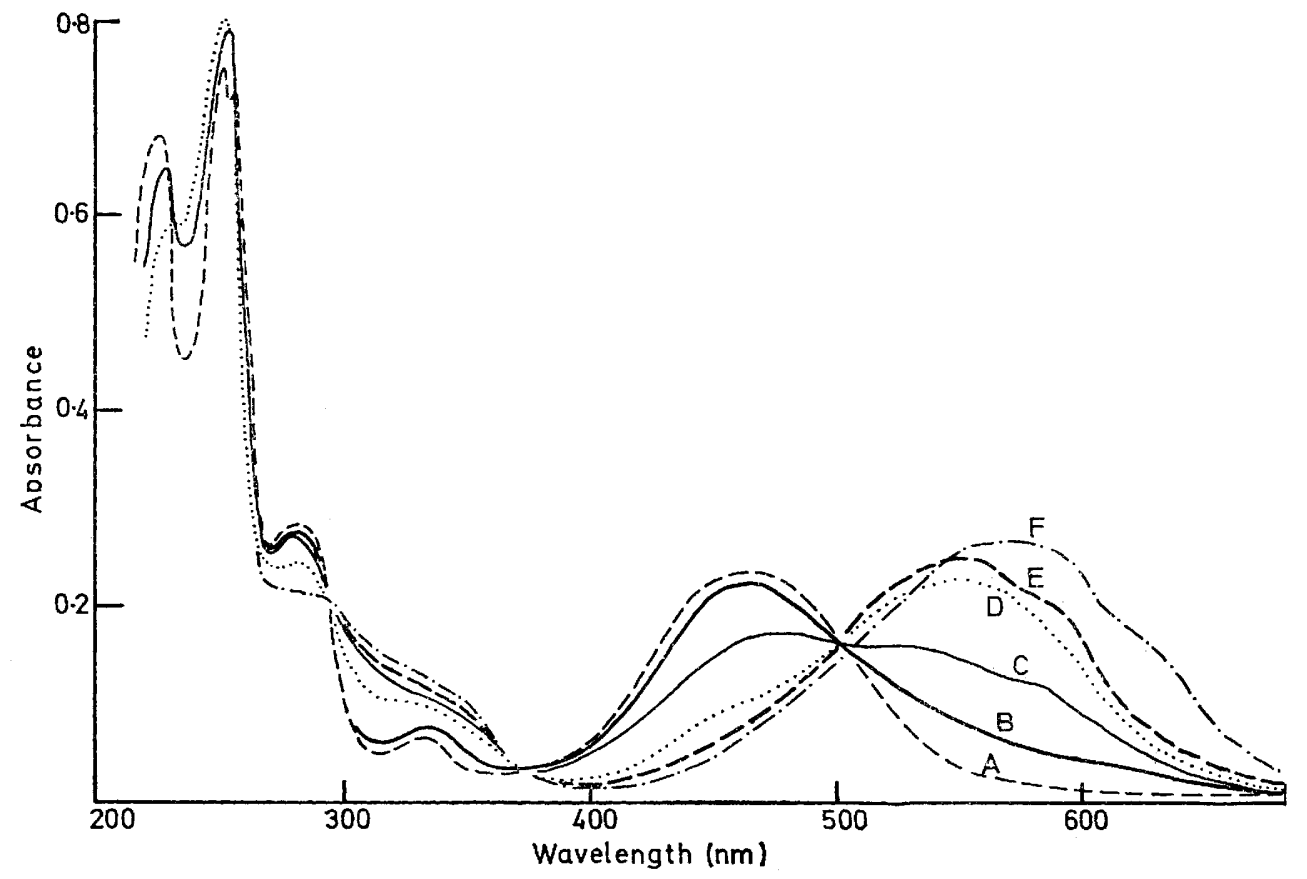

FIGURE 3 Absorption spectra of 1,4-dihydroxyanthraquinone $\left(2 \cdot 00 \times 10^{-5} \mathrm{M}\right)$ at different $\mathrm{pH}$ in aqueous solution: $\mathrm{A}$, pH $6 \cdot 6$; $\mathrm{B}, 7 \cdot 40 ; \mathrm{C}, 8 \cdot 35 ; \mathrm{D}, 9 \cdot 10 ; \mathrm{E}, 10 \cdot 10$; and $\mathrm{F}, 11 \cdot 30$

spectra in carbon tetrachloride) in solvents of high polarity, probably since solvent interaction with the hydroxy-group facilitates charge-transfer to the anthraquinone nucleus.

The $207 \mathrm{~nm}$. band, of low intensity in the spectrum of quinonoid system in 1,4-dihydroxyanthraquinone will be reduced (Figure 3); dissociation of the 1-and 4-protons may lead to increased delocalisation.

The $326 \mathrm{~nm}$. band is probably a $\pi-\pi^{*}$ transition of the ethylenic system, since the $272 \mathrm{~nm}$. band is almost 
Phys. Org.

absent at high $\mathrm{pH}$, and, furthermore, the intensity of the carbonyl band is lower in the i.r. spectra of the mono- and di-sodium salts of 1,4-dihydroxyanthraquinone, whereas the ethylenic band is not affected.

The existence of mesomeric structures may cause splitting of the benzonoid $\pi-\pi^{*}$ transition at $252 \mathrm{~nm}$.

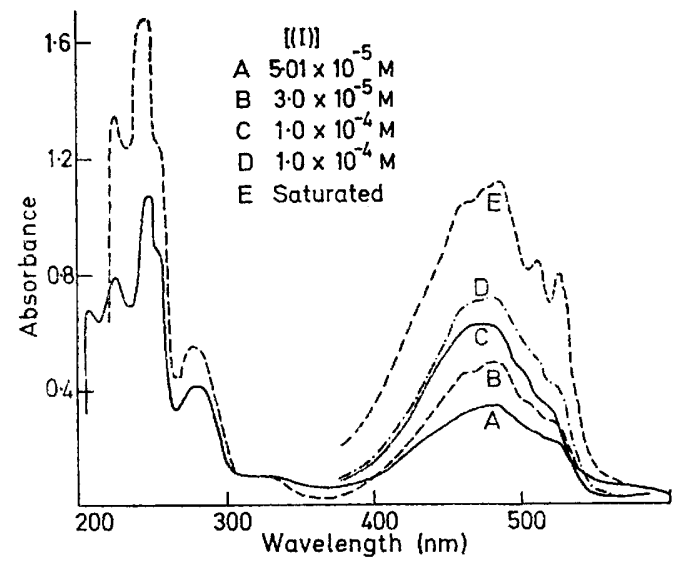

FIGURE 4 Absorption spectra of 1,4-dihydroxyanthraquinone (I) in ethanol (A), ether (B), acetone (C), chloroform (D), and carbon tetrachloride (E)

(Figure 4) and, for example, the three bands in the spectrum of 1,4-dihydroxyanthraquinone may be due to the structures (B)-(D).

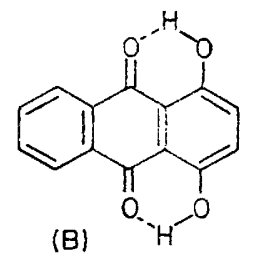

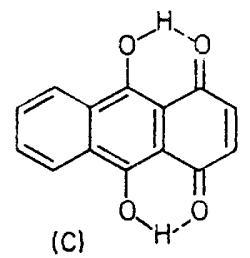<smiles>O=C1C=CC2=C3C(=c4ccccc4=C1O)OCOC3O2</smiles>

The spectra of the hydroxyanthraquinones in nonpolar solvents show vibrational structure, which is decreased or absent in polar solvents, possibly owing to solvation.

The absorption spectra in the visible region show a single, intense, charge-transfer band (Table 2).

TABLE 2

$\lambda_{\max }$ (nm.) of the charge-transfer bands in different solvents

\begin{tabular}{cccccc}
\multicolumn{5}{c}{ Solvent } \\
$\begin{array}{c}\text { Carbon } \\
\text { tetra- } \\
\text { Compound } \\
\text { chloride }\end{array}$ & Ethanol & Acetone & Ether & $\begin{array}{c}\text { Chloro- } \\
\text { form }\end{array}$ \\
(III) & 430 & 555 & 430 & 430 & 410 \\
& & & & & 420 \\
(I) & 475 & 475 & 475 & 475 & 475 \\
(II) & 425 & 425 & 420 & 425 & 425 \\
(IV) & 480 & 525 & $\mathbf{4 8 0}$ & $\mathbf{4 8 0}$ & 480 \\
(V) & 485 & $\mathbf{5 6 0}$ & $\mathbf{4 8 5}$ & $\mathbf{4 8 5}$ & $\mathbf{4 8 5}$
\end{tabular}

Beer's law applied in all cases, indicating that solutesolute interactions were of minor importance compared with solute-solvent interactions (Figure 5) for the range of concentrations used.
The presence of an intermolecular hydrogen bond is further supported by the spectra in mixed solvents containing a nonpolar component (e.g. carbon tetrachloride)
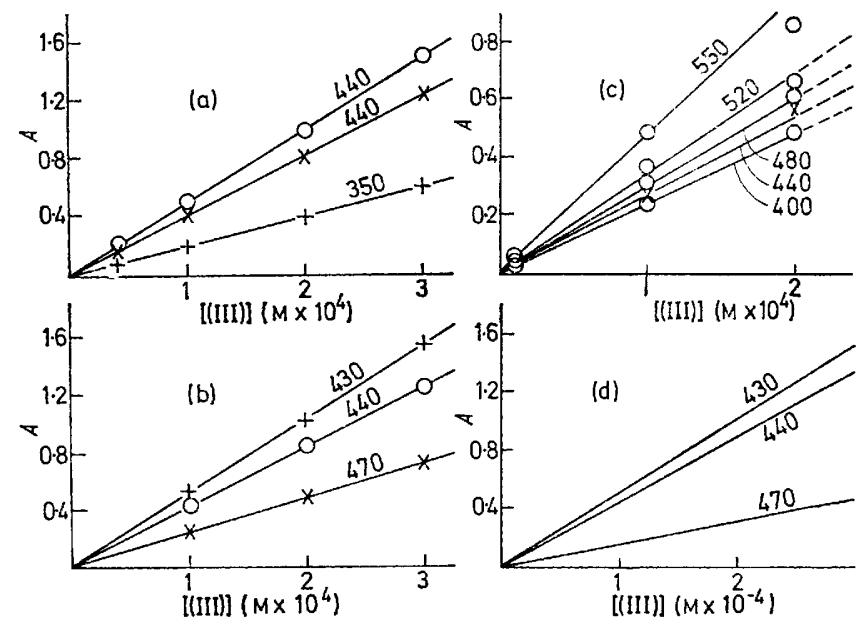

FIGURE 5 Variation of absorbance with concentration of 1,2dihydroxyanthraquinone (III) in different solvents at different wavelengths (wavelengths in $\mathrm{nm}$.); (A) in ether; (B) acetone, (C) ethanol, and (D) chloroform

and a proton acceptor (e.g. ethanol). Isobestic points were usually obtained when the concentration of the proton acceptor was varied at constant solute concentration
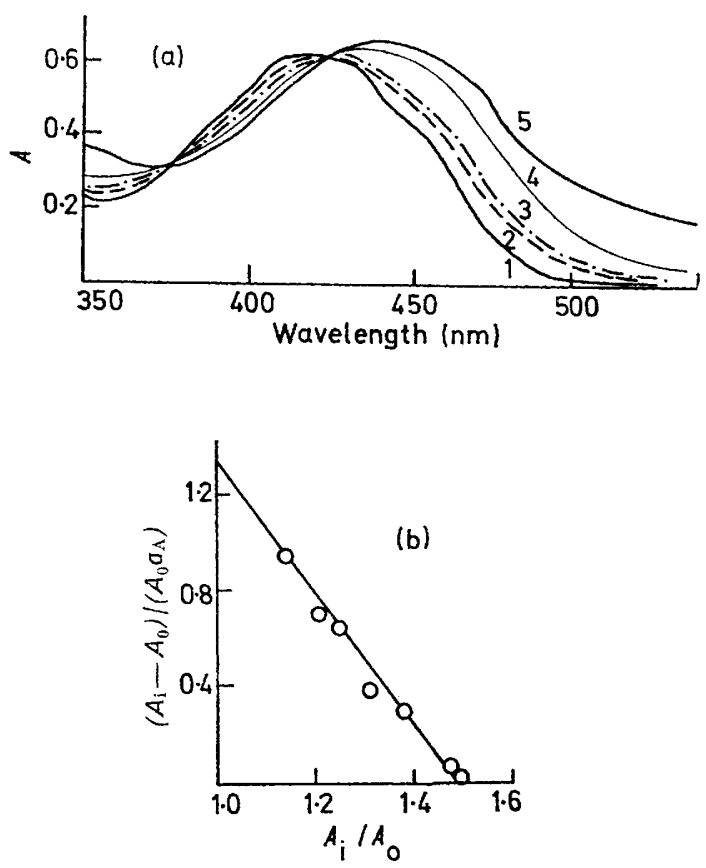

FIGURE 6 (a) Absorption spectra of 1,2-dihydroxyanthraquinone $\left(1 \times 10^{-4} \mathrm{M}\right.$-solution in carbon tetrachloride) for different concentrations of ethanol, and (b) plot of $\left(A_{1}-A_{0}\right) /$ $\left(A_{0} a_{A}\right)$ for 1,2-dihydroxyanthraquinone at $450 \mathrm{~nm}$.

(Figure 6), which indicates the existence of equilibrium (1) where $D$ and $A$ are the donor and acceptor, respec-

$$
\mathrm{D}+\mathrm{A} \rightarrow \mathrm{DA}
$$


tively, and DA is a $1: 1$ complex. The stability constant is given by equation (2).

$$
K=[\mathrm{DA}] /[\mathrm{D}] \cdot[\mathrm{A}]
$$

The effective concentrations of both $A$ and $D$ are given by equations ( $3 a$ and $b$ ) where the concentrations referred

$$
\begin{aligned}
& a_{\mathrm{A}}=[\mathrm{A}]+[\mathrm{DA}] \\
& a_{\mathrm{D}}=[\mathrm{D}]+[\mathrm{DA}]
\end{aligned}
$$

to are those at equilibrium.

The absorption at a given wavelength in a mixed solvent $\left(A_{\mathrm{i}}\right)$ is given by equation $(4)$, where $\varepsilon_{0}$ and $\varepsilon_{\mathrm{i}}$ are molar extinction coefficients of $\mathrm{D}$ and $\mathrm{DA}$, re-

$$
A_{\mathrm{i}}=l \varepsilon_{0}[\mathrm{D}]+l \varepsilon_{1}[\mathrm{DA}]
$$

spectively, and $l$ is the path length. The absorbance in an inert solvent $\left(A_{0}\right)$ is given by equation (5).

$$
A_{0}=l \varepsilon_{0} a_{\mathrm{D}}
$$

Equation (6) can be derived from equations $(2)-(5)$ :

$$
\left(A_{\mathrm{i}}-A_{0}\right) /\left(A_{0} a_{\mathrm{A}}\right)=K \varepsilon_{1} / \varepsilon_{0}-K A_{\mathrm{i}} / A_{0}
$$

If these assumptions are correct a plot of $\left(A_{\mathrm{i}}-A_{0}\right)$ ( $A_{0} a_{\mathrm{A}}$ ) against $A_{\mathrm{i}} / A_{0}$ should be linear, with slope $-K$ and intercept $K \varepsilon_{1} / \varepsilon_{0}$. Application of equation (6) to the 1,2-dihydroxyanthraquinone-ethanol system confirmed that a $1: 1$ complex was formed, with a stability constant of $2 \cdot 6$ (Figure 6 ). 